\title{
Structure, Defects and Magnetism of Electrospun Hematite Nanofibers Silica-Coated by Atomic Layer Deposition
}

\author{
Alessandro Ponti ${ }^{\dagger}, \bar{\delta}$, Muhammad Hamid Raza ${ }^{\#, \delta}$, Fabiola Pantò ${ }^{\ddagger}$, Anna Maria Ferretti ${ }^{\dagger}$, \\ Claudia Triolo $^{\S}$, Salvatore Patanèll, Nicola Pinna ${ }^{\#, *}$, Saveria Santangelo ${ }^{\S, *}$ \\ ${ }^{\dagger}$ Laboratorio di Nanotecnologie, Istituto di Scienze e Tecnologie Molecolari (ISTM), Consiglio Nazionale delle \\ Ricerche, Via G. Fantoli 16/15, 20138, Italy \\ \# Institut für Chemie and IRIS Adlershof, Humboldt-Universität zu Berlin, Brook-Taylor Str. 2, 12489 Berlin, \\ Germany \\ * Istituto di Tecnologie Avanzate per l’Energia (ITAE), Consiglio Nazionale delle Ricerche, Salita S. Lucia \\ Sopra Contesse 5, 98126 Messina, Italy \\ $\S$ Dipartimento di Ingegneria Civile, dell'Energia, dell'Ambiente e dei Materiali (DICEAM), Università \\ "Mediterranea", Loc. Feo di Vito, 89122 Reggio Calabria, Italy \\ " Dipartimento di Scienze Matematiche e Informatiche, Scienze Fisiche e Scienze della Terra (MIFT), Università \\ di Messina, Viale Stagno d'Alcontres 31, 98166 Messina, Italy
}

Number of pages: 20

Number of figures: 18

Number of schemes: 0

Number of tables: 2 
(a)

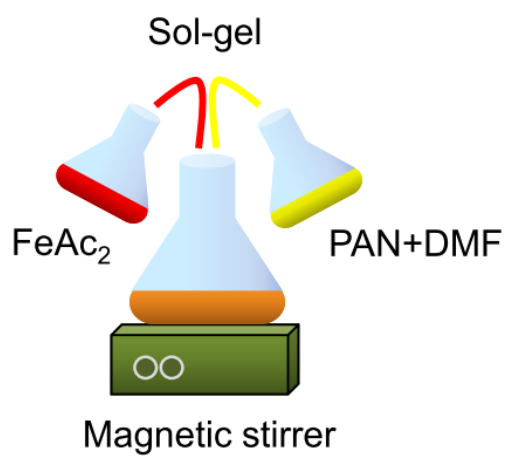

(b)

$$
\mathrm{FeAc}_{2}+\mathrm{PAN}+\mathrm{DMF}
$$
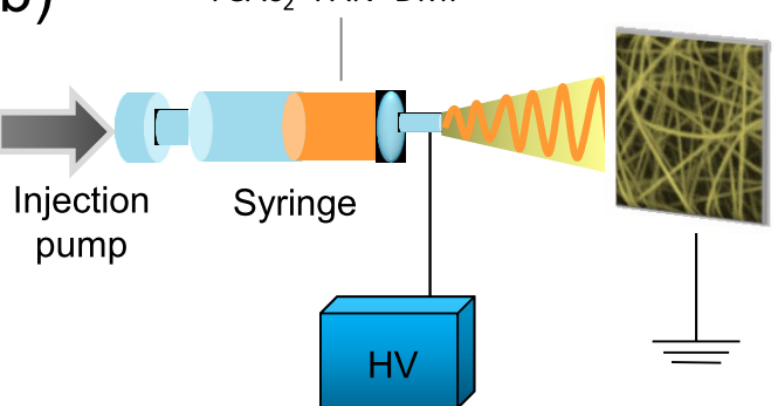

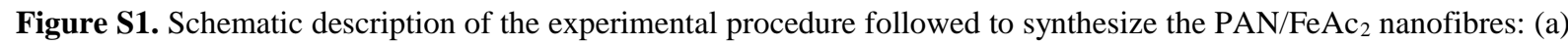
preparation of the precursor solution by sol-gel method, (b) electro-spinning.

\section{Preparation of the $\mathrm{SiO}_{2}$-Coated $\alpha-\mathrm{Fe}_{2} \mathrm{O}_{3}$ Nanofibers}



Figure S2. Sketch of the experimental setup utilised for the ALD process and (encircled) mechanistic scheme of the reaction steps in one cycle $\mathrm{SiO}_{2}$ ALD from APTES, inspired by ref. S1. 



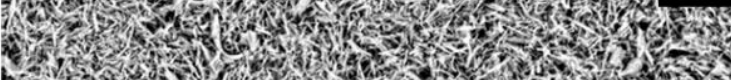

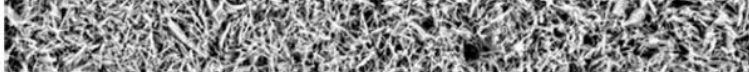

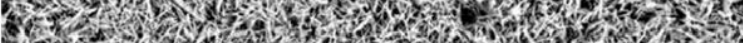

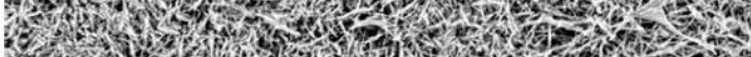

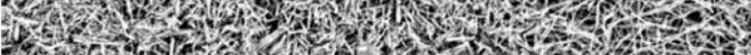

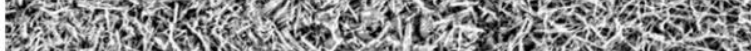
2.

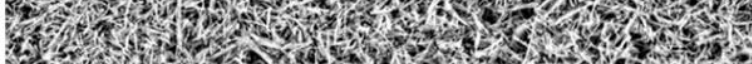

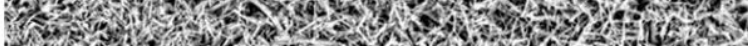
*.16. I

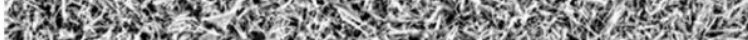

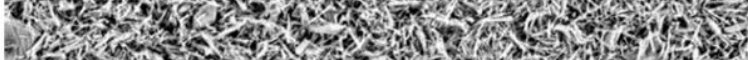

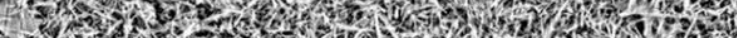

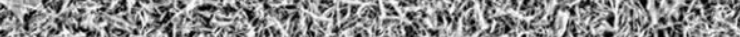

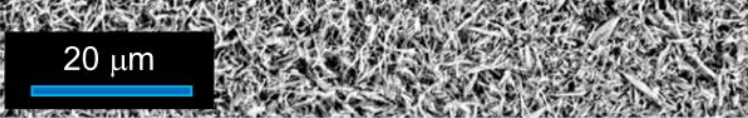
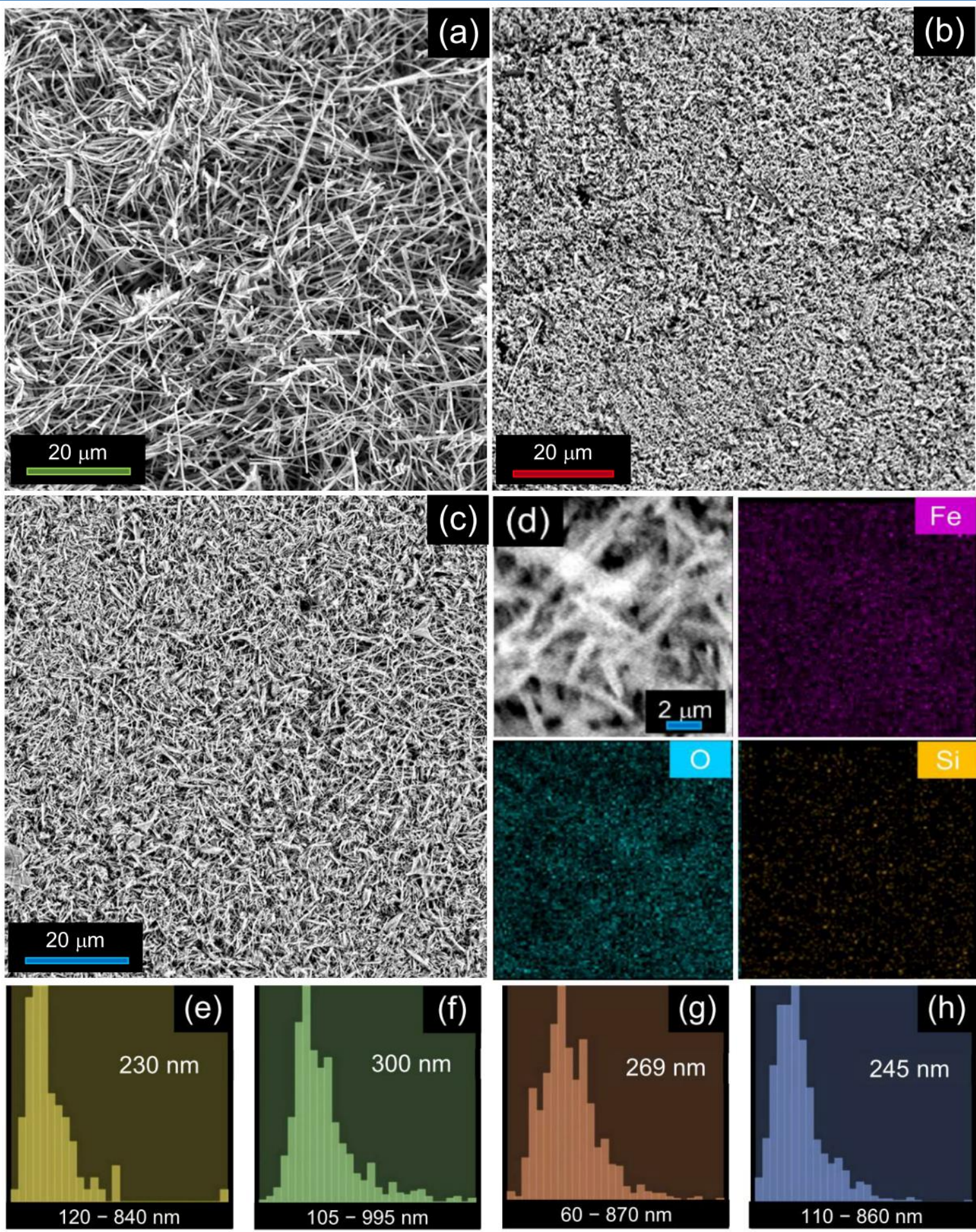

Figure S3. (a-c) Low-magnification SEM images, (d) EDX elemental mapping and (e-h) histograms of the diameters of the NFs. The shown images refer to samples (a) NF-25, (b) NF-25/600 and (c) NF-600/25; EDX maps refer to sample NF-600/25. Histograms of NF diameter refer to samples (e) NF-600, (f) NF-25, (g) NF-25/600 and (h) NF$600 / 25$ 

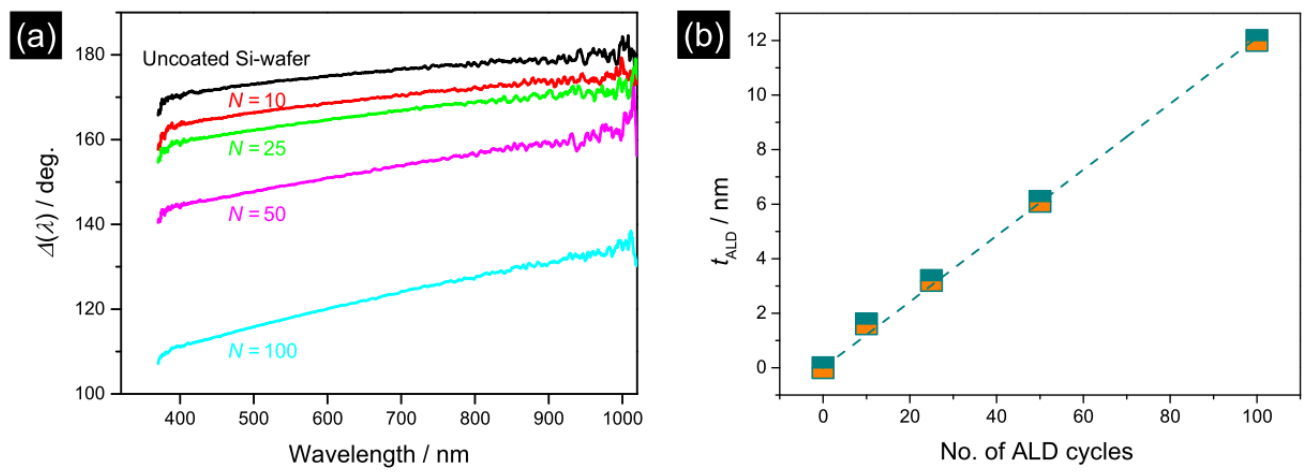

Figure S4. (a) Spectroscopic ellipsometry curves, $\Delta(\lambda)$ ), for the uncoated and silica-coated Si-wafers with various numbers of ALD cycles $(N)$. The systematic shift in the curves proves the increase in the film thickness $\left(t_{\text {ALD }}\right)$ with increasing $N$. (b) Linear fit to the data $\left(R^{2}=0.999\right)$.
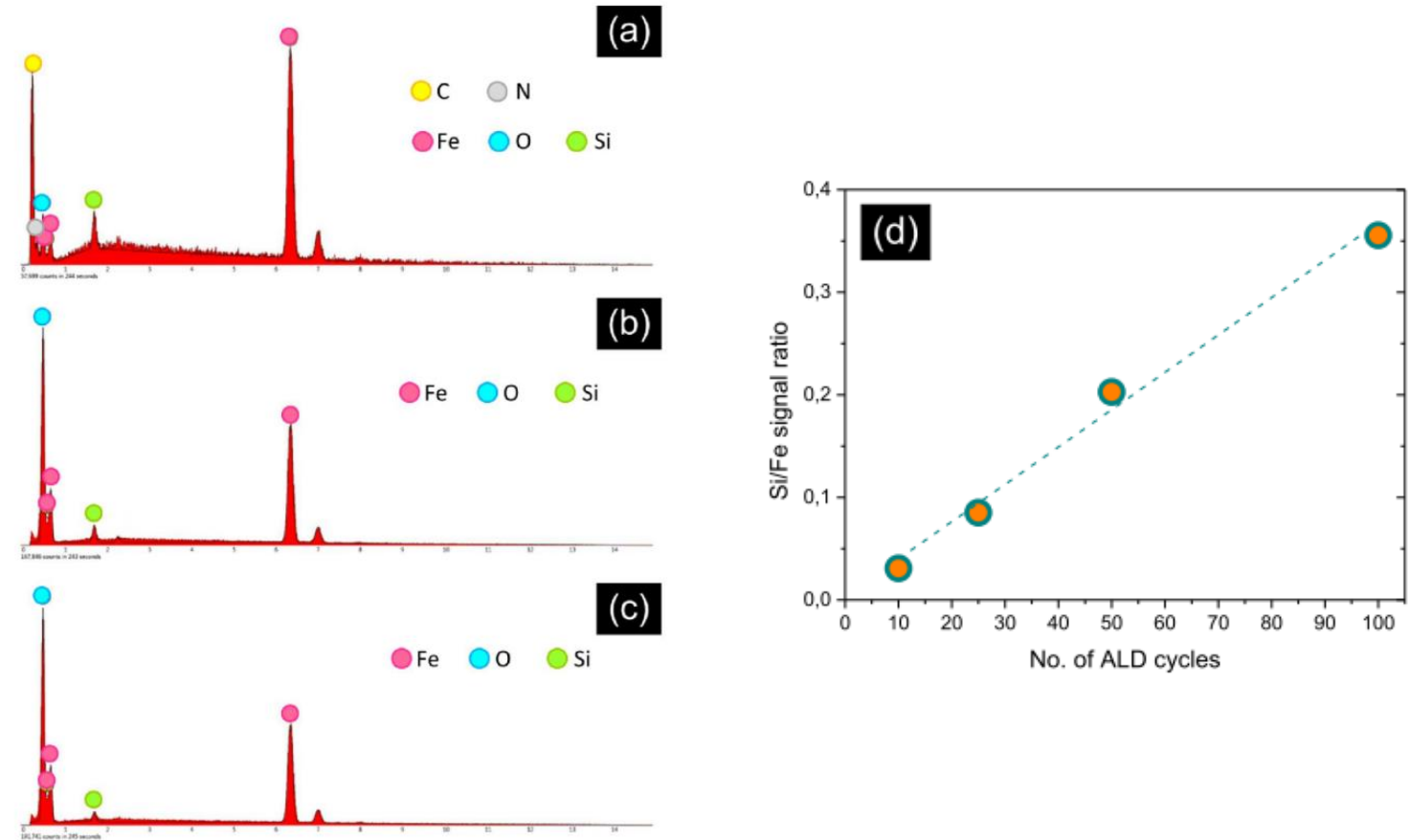

Figure S5. (a-c) SEM/EDX spectra of samples (a) NF-25, (b) NF-25/600 and (c) NF-600/25. Before the HT (a), the signals of $\mathrm{C}$ and $\mathrm{N}$, present in the organic component of the NFs, are detected. (d) $\mathrm{Si} / \mathrm{Fe}$ EDX signal intensity ratio as a function of the No. of ALD cycles. The shown data refer to samples NF-600/N. 


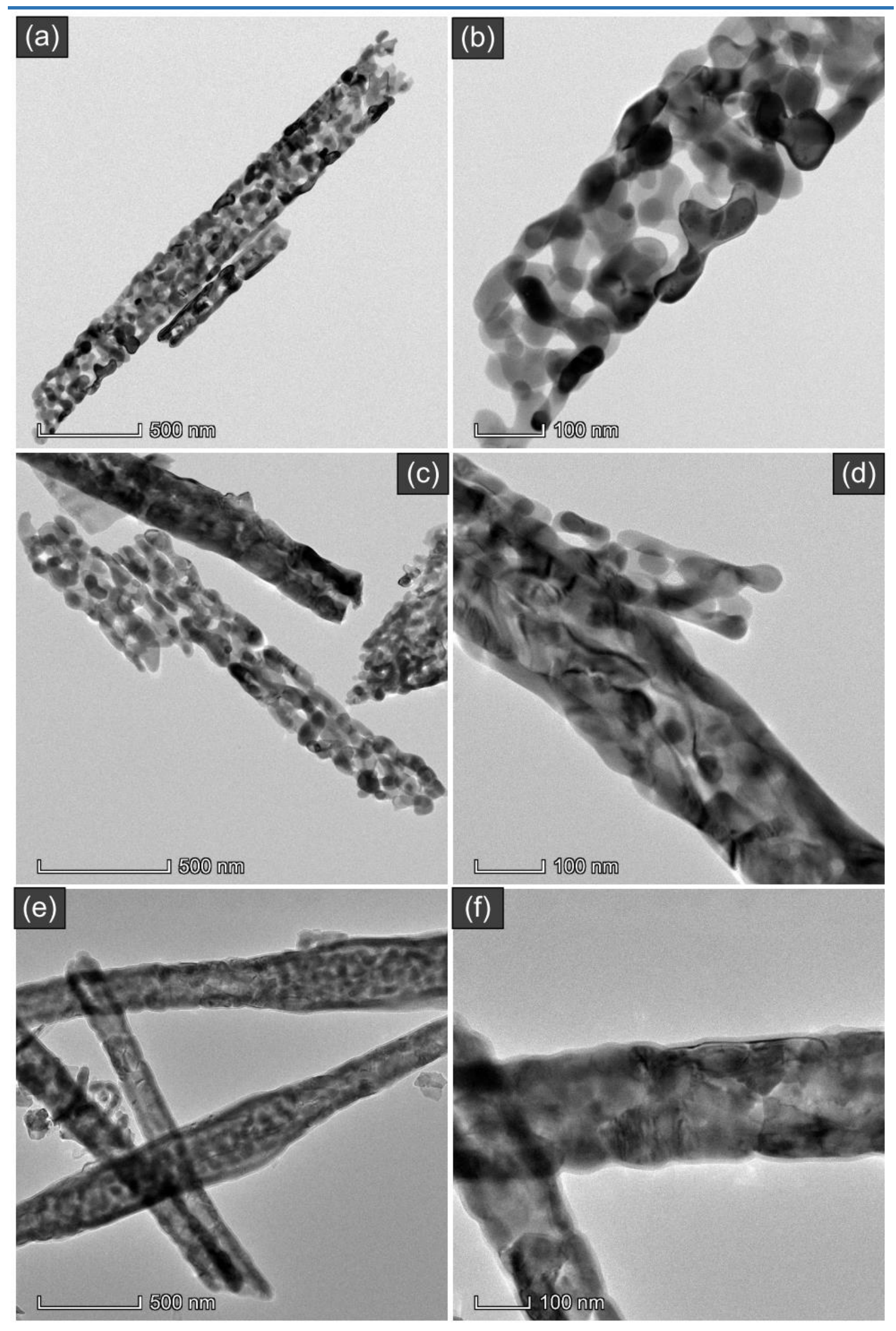




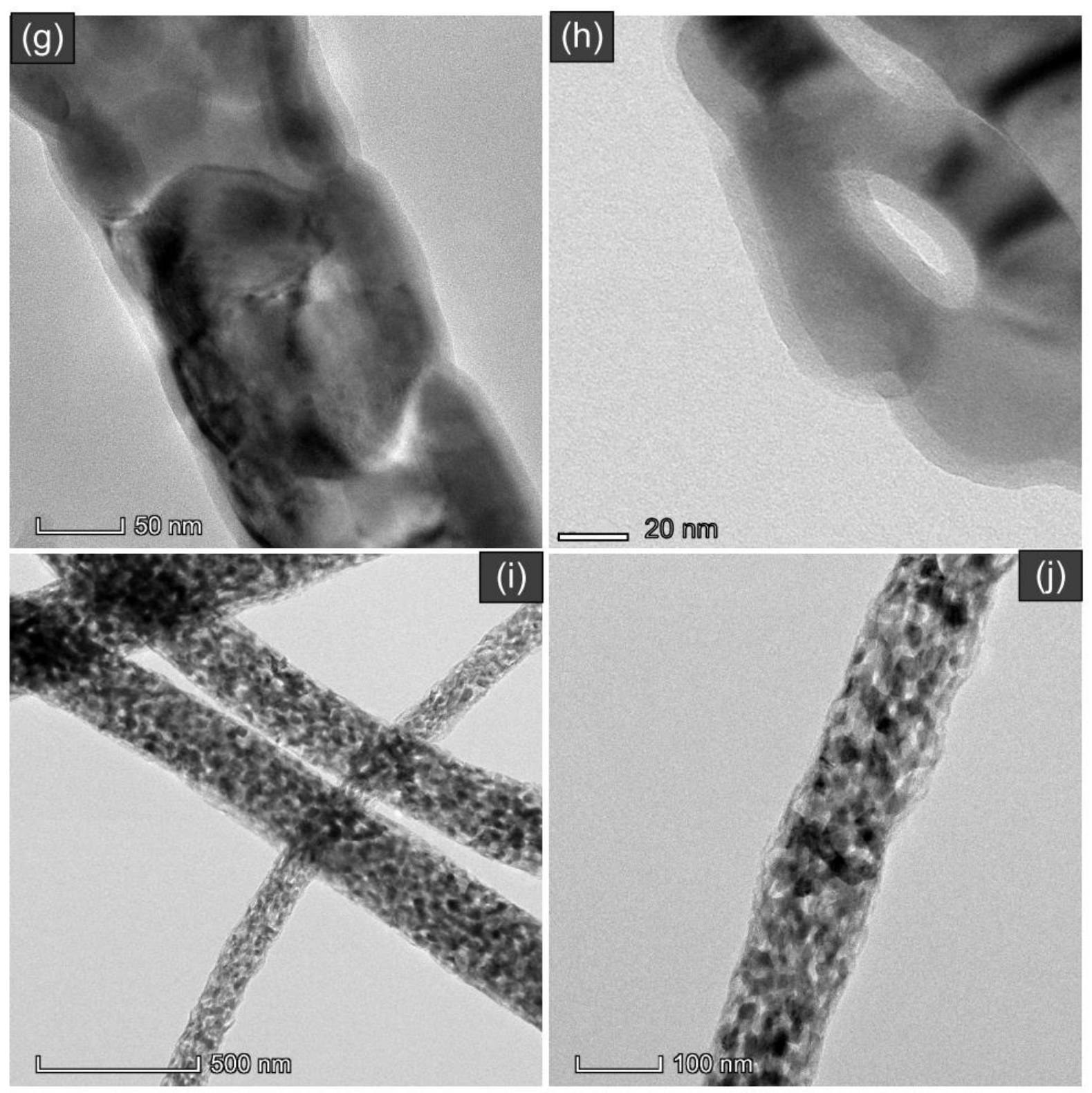

Figure S6. Morphology of the NFs, as monitored by TEM. The shown images refer to samples (a,b) NF-600, (c,d) NF600/25, (e-h) NF-600/100 and (i,j) NF-25/600.

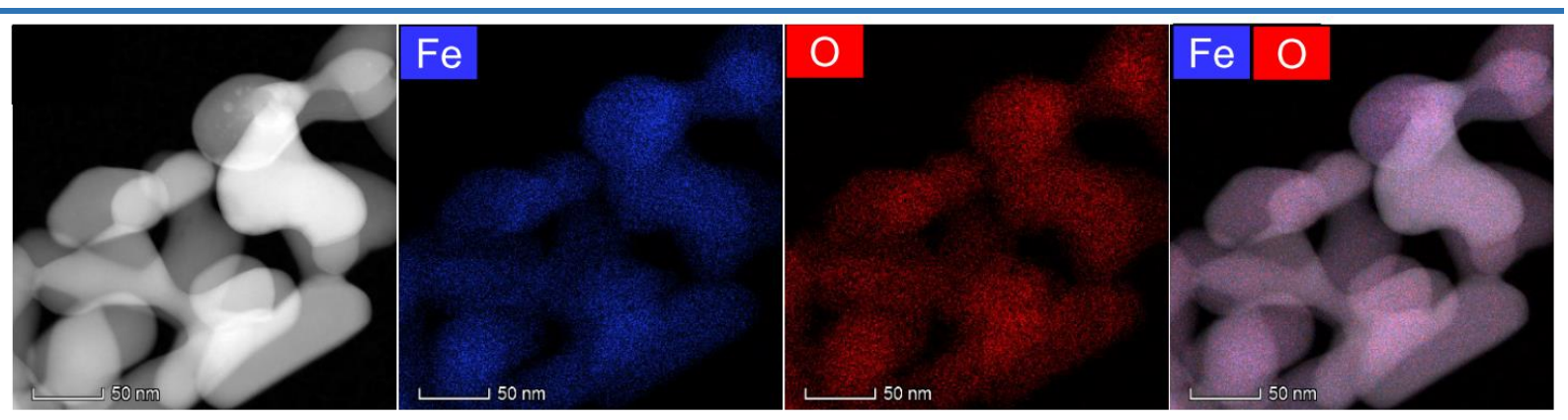

Figure S10. Elemental composition of the NFs in sample NF-600, as resulting from HRTEM/EDX analysis. 


\section{Geometrical Phase Analysis of HRTEM Images}

The geometrical phase analysis (GPA) of HRTEM crystal fringes proposed by $\mathrm{Hÿtch}^{\mathrm{S} 2}$ provides information about the crystal lattice and defects of crystallites . In the present case, the analysis intends to answer to the following questions: (i) Are the hematite grains visible in the (S)TEM images of the NFs single-crystals or polycrystalline? (ii) Is the lattice orientation of neighboring crystallites and grains similar? (iii) What are the characteristics of the crystallite and grain lattice strain?

We use GPA to analyze HRTEM images and seek answers to the questions above. It should be kept in mind that this method has been developed for and usually applied to thin films. HRTEM images of thin films have good quality (the film is thin and its thickness constant in many cases), the film is highly crystalline with few defects and relatively simple interfaces, and tilting is used to achieve simple zone axis alignment. In the case of crystallites, one must be cautious in interpreting results since the amplitude depends strongly on thickness, especially for strong reflections, and superimposed crystallites may give rise to peculiar effects, such as moiré. In our case, additional difficulty arises from (i) the large size (= thickness) of the crystallites, which are estimated to be around $20-30 \mathrm{~nm}$, well above the best thickness for HRTEM work (a few nm); and (ii) the large size of the grains and of the NFs themselves, which leads to widespread overlap of crystallites.

The procedure can be briefly summarized as follows. HRTEM images with clearly visible lattice fringes are Fourier transformed (FT-ed). Inspection of the 2D FT-ed image in the reciprocal space ( $k$-space) may reveal peaks corresponding to vectors $g_{h k l}$ of the reciprocal crystal lattice provided that the crystallite(s) are suitably oriented for effective coherent scattering. The position of these peaks allows one to find out the Miller indices of the involved $h k l$ lattice plane. Inspection of the shape of the $k$-space peak provides information about the number and quality of the crystallites contributing to the peak. Note that reciprocal space peaks contain both kinematic scattering and dynamic scattering. The latter can be effective even in the absence of kinematic scattering, e.g., due to systematic absences.

Next, the FT-ed image is masked with a 2D isotropic Gaussian filter usually (but not always) centered on the peak maximum and with width achieving the compromise of good spatial resolution (wide filter) and effective masking (narrow filter), i.e., exclusion of other peaks in the $k$-space. The filtered reciprocal space image is inverse FT-ed back to real space yielding a complex real-space image. The amplitude $\left(\operatorname{Re}^{2}+\mathrm{Im}^{2}\right)^{1 / 2}$ of this image is proportional to the amplitude of the crystal fringes. The amplitude image shows the shape of the crystallite(s) and provides some information about the lattice defects. It must however be interpreted with caution (see above). The background of the amplitude image (i.e. where the crystal fringes are not present) is dark (black).

The amplitude-colored image is the original $\mathrm{B} / \mathrm{W}$ image after colorizing with yellow hue the regions where the crystal fringes are present. The color saturation is proportional to the fringe amplitude while the image lightness is left 
unchanged (HSL color system used). The amplitude-colored image helps in putting the crystallite(s) displaying the investigated fringes in the context of the other crystallites of the imaged region of the NF.

The phase $[\arctan (\mathrm{Im} / \mathrm{Re})]$ of the complex real-space image portrays the phase of the crystal fringes. The phase image also shows the shape of the crystallite(s), often in a less effective way than the amplitude image does, but provides in-depth information about the lattice defects. The phase image of a perfect crystal has constant color (= constant phase) so the phase image of a perfect single crystallite is a patch of constant gray. The background of the phase image (i.e. where the crystal fringes are not present) resembles pure noise because phase is ill-defined here.

A striped appearance of a crystallite means that the crystallite fringes have a reciprocal space $g_{h k l}$ vector that is displaced from the mask (filter) center. If another crystallite is well centered (flat colored patch in the real-space image), the striped appearance is evidence of relative misalignment (the $g_{h k l}$ vectors have equal magnitude but different orientation) or unequal interplanar distance (the $g_{h k l}$ vectors have equal orientation but different magnitude, i.e., they are radially displaced) or both.

Rapid phase (= color) variation within a crystallite is evidence of the presence of lattice disorder either by misalignment or unequal cell size or both, as above. If rapid phase change occurs on a line (straight or curved), this may mark the boundary between two crystallite. Dislocations seen head-on display a typical pattern in phase images consisting of a point where a $180^{\circ}$ phase change occurs in a tiny distance. This feature are a hallmark of crystal dislocations.

From the phase of the complex real-space image, information about lattice strain and rotation can be gained. This is usually carried out using two non-aligned peaks $g_{h k l}$ and $g_{h^{\prime} k^{\prime} l^{\prime}}$, as pioneered by Hÿtch. Rouviére and Sarigiannidou later showed how strain and rotation can be calculated using a single $g_{h k l}$ peak. ${ }^{\mathrm{S} 3}$ In this case however, Lagrange strain is only calculated along $g_{h k l}, i . e$, perpendicular to the lattice fringes. Strain and rotation images can be obtained using Eq.'s 60 and 61 in ref. S3. The phase $\arctan (\mathrm{Im} / \mathrm{Re})$ of the complex image was processed to produce the lattice rotation and Lagrange strain maps. Both rotation and strain are relative to the reference lattice generated by $\mathbf{g}_{h k l}$. The GPA results below are presented in three separate images: (i) the fringe-intensity-colored TEM image, i.e. the original black-and-white HRTEM image colorized with yellow hue proportional to the lattice fringe amplitude; (ii) the rotation image, expressed as rotation in degree from $\mathbf{g}_{h k l}$; (iii) the Lagrange strain image, expressed as percentage deviation of the interplanar distance with respect to the reference distance $1 / g_{h k l}$. The intensity of the lattice fringes in a grain often displays strong oscillations caused by the moiré effect.

Below are presented some additional GPA results in three separate images: (i) the fringe-intensity-colored TEM image, i.e. the original black-and-white HRTEM image colorized with yellow hue proportional to the lattice fringe amplitude; (ii) the rotation image, expressed as rotation in degree from $\mathbf{g}_{h k l}$; (iii) the Lagrange strain image, expressed as percentage deviation of the interplanar distance with respect to the reference distance $1 / g_{h k l}$. In the SI file "Analysis of HRTEM", other GPA results are presented. There, six images per analysis are shown, comprising the three just 
described images augmented with (iv) the FT-ed image with the mask outline, (v) the amplitude $\left(\operatorname{Re}^{2}+\operatorname{Im}^{2}\right)^{1 / 2}$ image, and (vi) the phase $\arctan (\mathrm{Im} / \mathrm{Re})$ image.

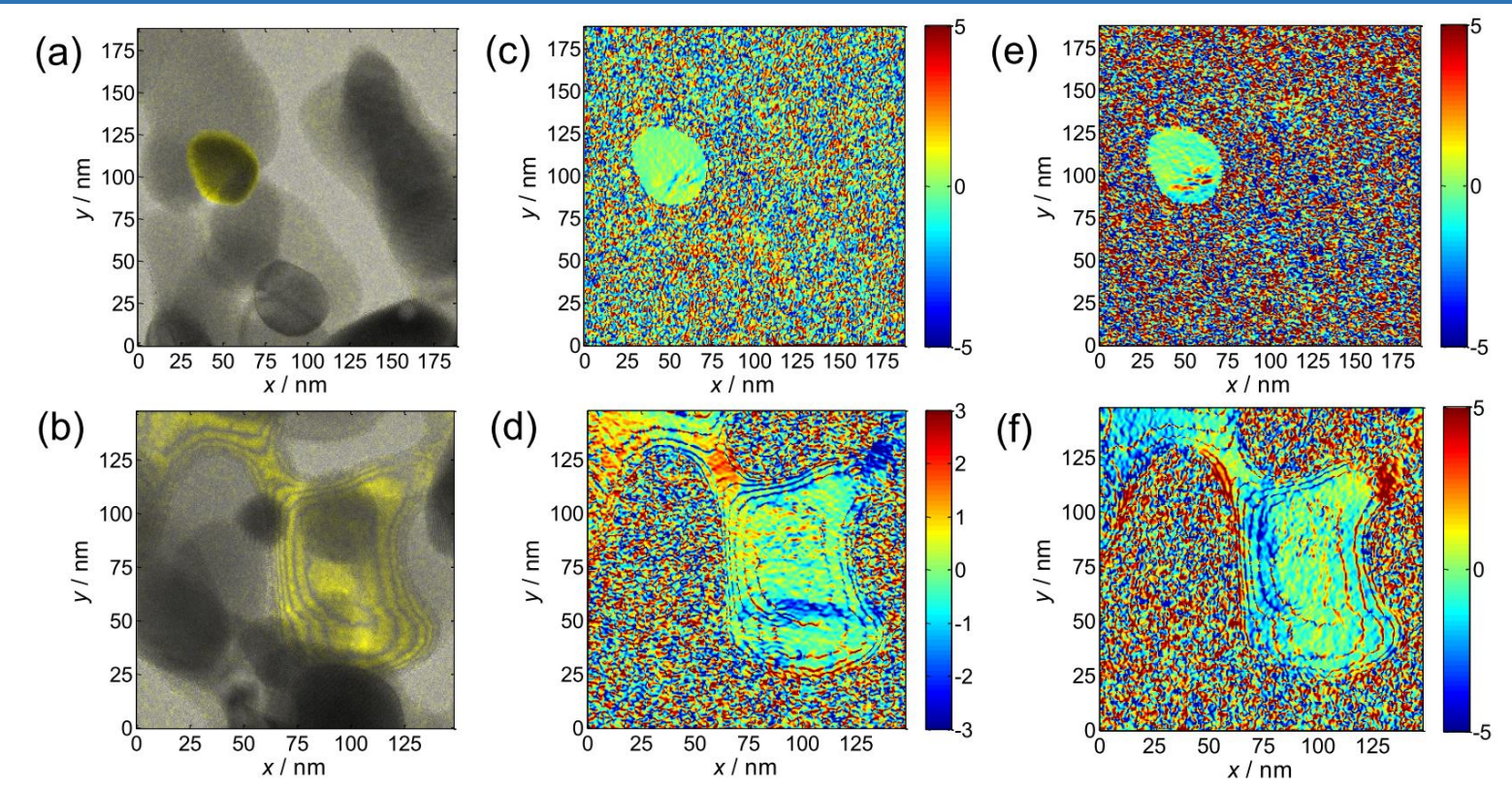

Figure S7. Geometrical phase analysis of the NFs in sample NF-600. (a-b) Intensity-colorized maps, (c-d) lattice rotation maps $\left(^{\circ}\right)$ and (e-f) lattice strain maps (\% cell size). Top row: SC grain , <104> lattice fringes; bottom row: QSC grain, <104> lattice fringes.
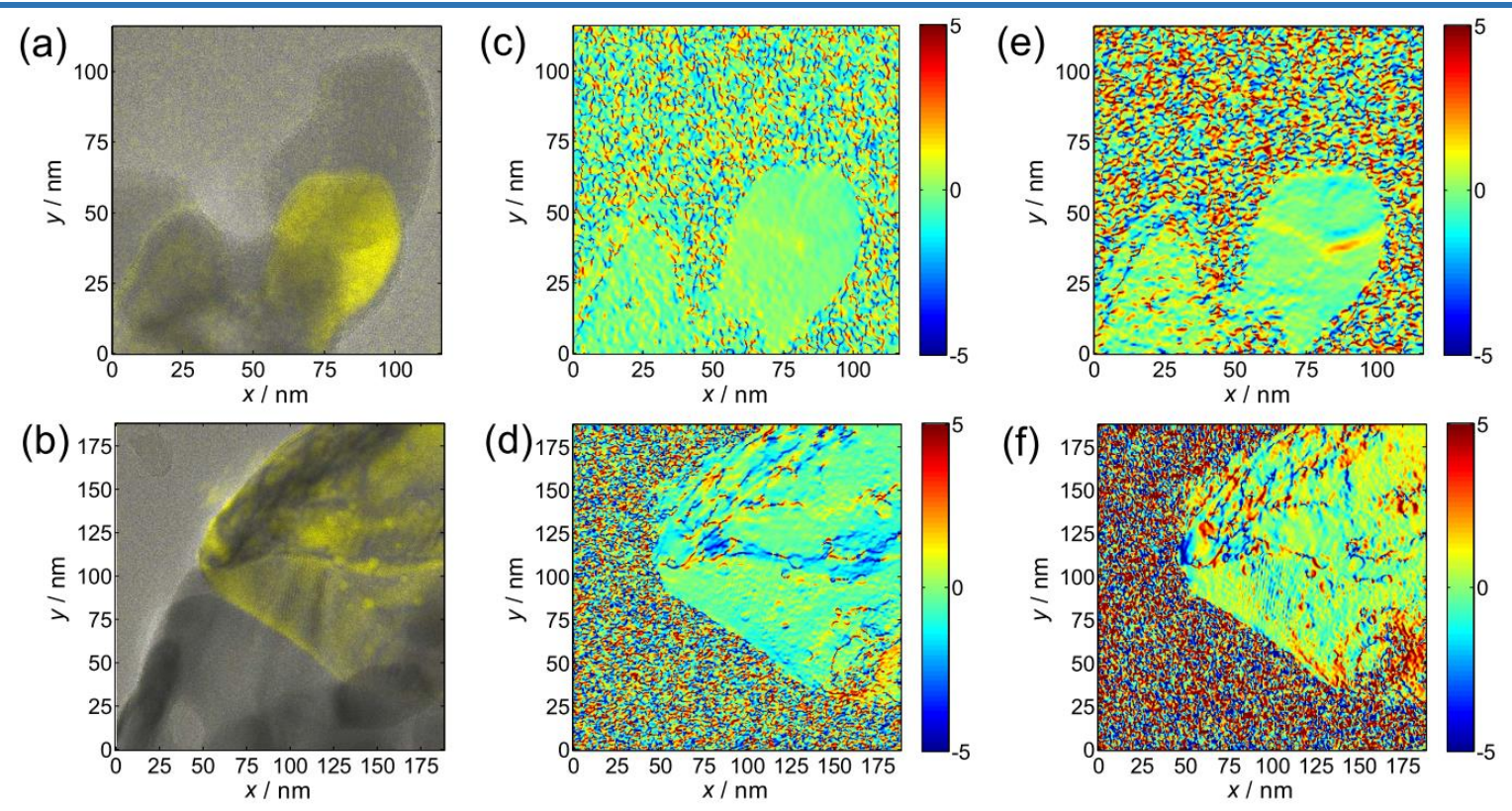

Figure S8. Geometrical phase analysis of the NFs in sample NF-600/25. (a-b) Intensity-colorized maps, (c-d) lattice rotation maps $\left({ }^{\circ}\right)$ and (e-f) lattice strain maps (\% cell size). Top row: SC grain, <122> lattice fringes; bottom row: SSC grain, <113> lattice fringes. 

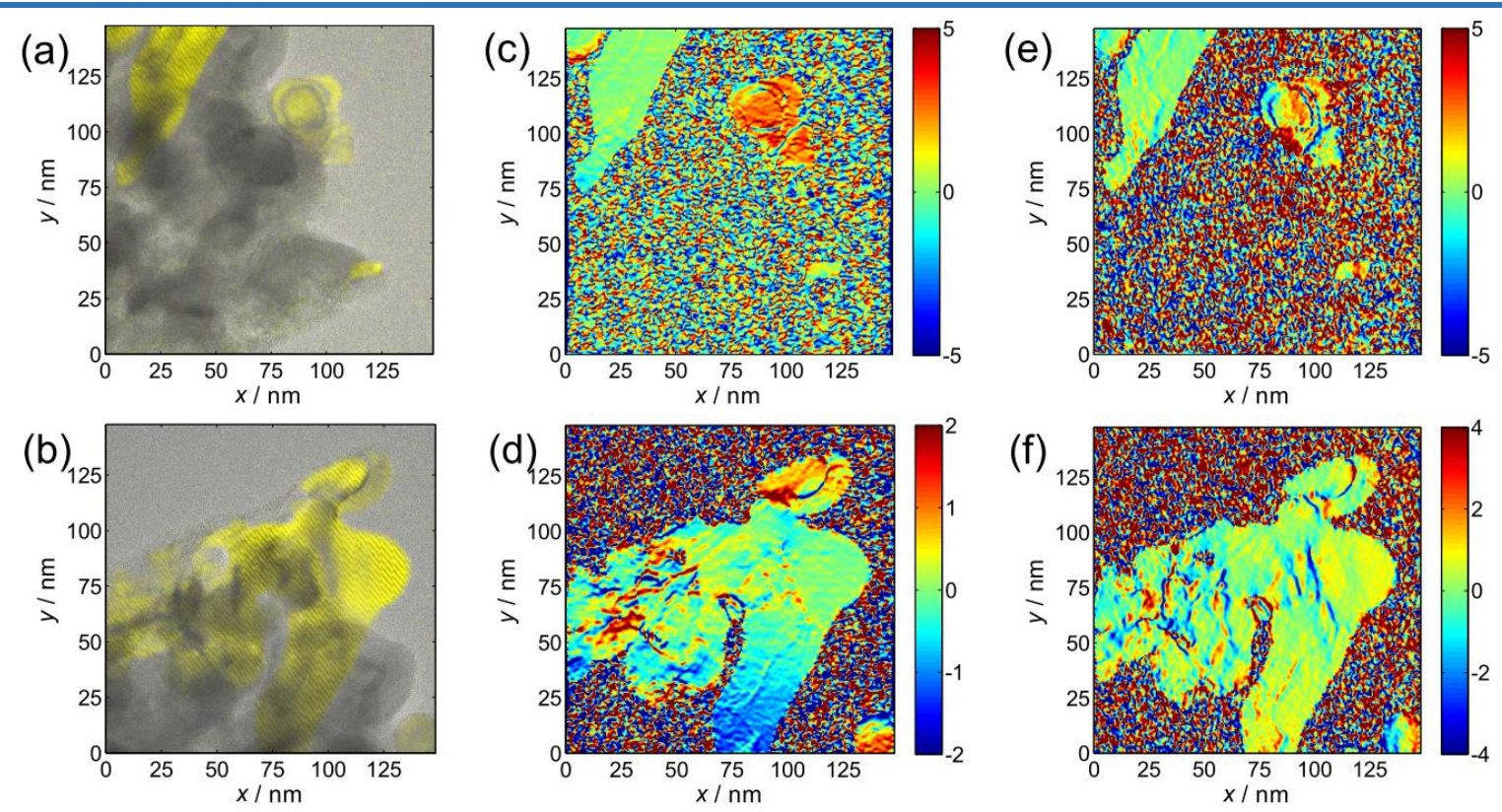

Figure S9. Geometrical phase analysis of the NFs in sample NF-25/600. (a-b) Intensity-colorized maps, (c-d) lattice rotation maps $\left({ }^{\circ}\right)$ and (e-f) lattice strain maps (\% cell size). Top row: <104> lattice fringes highlight a large SC grain (top left), a large QSC grain (top right), and a small SC grain (bottom right); bottom row: SC grain, <012> lattice fringes. 


\section{Projection Analysis of (S)TEM Images and of EDX Maps}

The analysis intends to answer to the following questions:

1. Are the nanofibers (NFs) solid or hollow?

2. How is the silica distributed in the NFs?

Since the NFs are not completely solid and their "porous" skeleton is constituted by interconnected crystalline grains, one may wonder whether the NFs are solid (circular cross-section) or hollow (annular cross-section) and if silica forms just an external layer or it is also present within the NF. Projection analysis of TEM and HAADF-STEM images and STEM-EDX maps of $\mathrm{Fe}, \mathrm{Si}$, and $\mathrm{O}$ allowed us to answer both questions above. In particular, both (S)TEM images and EDX maps can reveal the inner morphology of the NFs, while only STEM-EDX maps can shed light on both the Si distribution and the inner morphology of the NFs.

We attacked this problem by a two-step procedure. After finding an image/map with not too large scale, (1) a straight, constant-diameter NF (or region of a NF) is selected and integrated (projected) along the NF axis; (2) next, the projected intensity is fitted to models appropriate for solid or hollow NFs. We implemented this procedure as Matlab programs. The EDX maps are ideally suited to this approach since the map intensity is proportional to the mass density. STEM HAADF images are also well suited to answer to question 1 despite the fact that HAADF intensity is proportional to atomic number density weighted by $Z^{2}$, as opposed to mass density $=$ atomic number density weighted by $A$. In our case, the HAADF signal is biased toward hematite with respect to the EDX signal. Conventional TEM images are usually not suited to the present approach since their intensity depends on both mass and diffraction (Bragg) contrast. In the present case, the large size of the NFs (diameter 150-300 nm) makes mass contrast more important than in the usual thin-sample case, and the polycrystallinity of the NFs makes the Bragg contrast more spatially homogeneous than in the thin-foil or single-nanocrystal cases. Though not ideal, we use conventional TEM images to support conclusions drawn from the analysis of EDX maps and STEM HAADF images.

As anticipated, the projection analysis of images of NFs consists in (1) selecting the image of a straight, constantdiameter NF (or a region of a NF) and (2) integrating (project) the image intensity along the NF axis. Representative examples of straight, constant-diameter NFs (or NF regions) selected for projection analysis are depicted in Figure S11. The resulting 1D transverse projection is then fitted to models appropriate for solid or hollow NFs:

$$
\begin{aligned}
& I_{\text {solid }}=I_{\text {background }}+I_{0} \sqrt{1-(r / R)^{2}} \\
& I_{\text {hollow }}=I_{\text {background }}+I_{0}\left(\sqrt{1-\left(r / R_{\text {out }}\right)^{2}}-\sqrt{1-\left(r / R_{\text {in }}\right)^{2}}\right)
\end{aligned}
$$

where $I_{\text {background }}$ is the projected background intensity, $I_{0}$ is a constant depending on the NF size and composition, $r$ is the distance from the NF axis, $R$ is the radius of a solid NF, and $R_{\text {out }}$ and $R_{\text {in }}$ are the outer and inner diameter of a hollow NF, respectively. Note that these models assume that the NF have constant density when averaged over the 
projected NF segment. We projected NF segments longer than a few hundreds nm. Comparison of these models with the experimental projection provides information on the internal NF morphology.

In the SI file “Analysis of TEM_STEM_EDX”, other results of the projection analysis are presented.
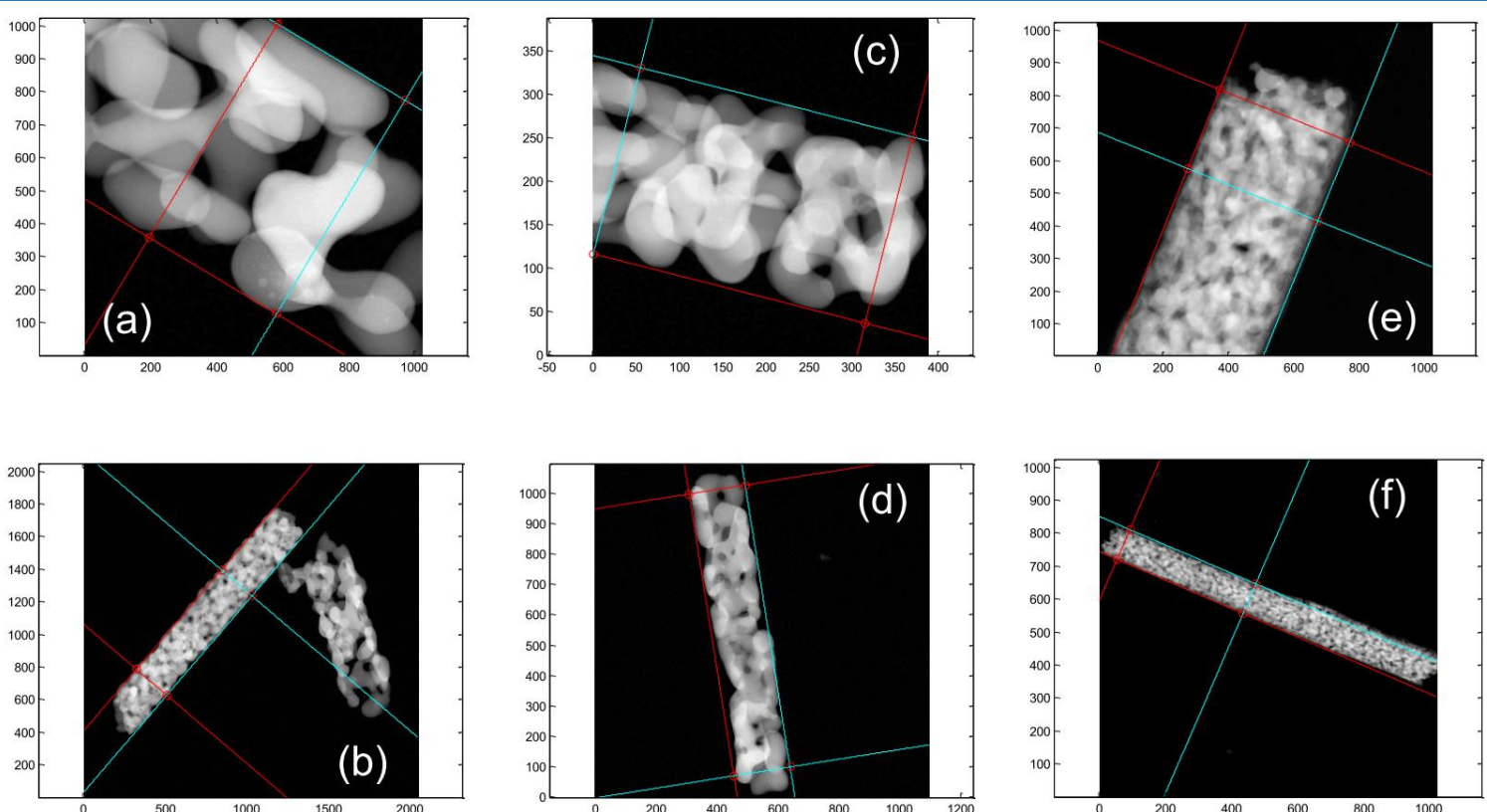

Figure S11. Representative examples of straight, constant-diameter NFs (or NF regions) from samples (a,b) NF-600, (c,d) NF-600/25 and (e,f) NF-25/600 that were selected for projection analysis. 


\section{Williamson-Hall Analysis}

The powder XRD data were utilized to calculate the peak broadening with crystallite size and lattice strain due to the dislocation. The size of the particles $\left(d_{\mathrm{WH}}\right)$ and strain $\left(\varepsilon_{\mathrm{WH}}\right)$ were estimated by applying the Williamson-Hall (WH) considering the uniform deformation model (UDM). The full width at half maximum (FWHM) was calculated by applying the Gaussian function on three main diffraction peaks, (104), (110) and (113), at the $2 \theta$-angles of $15.1^{\circ}, 16.2^{\circ}$ and $18.5^{\circ}$, respectively. The instrument-corrected broadening was utilized for the analysis. The breadth of the Bragg peak is a combination of both sample-dependent and instrumental effects. To de-convolute these contributions, the instrumental broadening was estimated by a diffraction pattern from the line broadening of a standard material (silicon).

A comprehensive description of the method is reported elsewhere. ${ }^{\text {S4-S6 }}$

Figure S12. (a-b) XRD patterns of the NFs at the (a) final and (b) early stages of their production (no peak attributable to iron oxide is detected in the NFs prior to calcination). (c) Williamson-Hall (WH) plots for the most representative samples. The correlation coefficient $\left(R^{2}\right)$ in the linear fits to the data is reported.

\begin{tabular}{ccc}
\hline Table S1. WH crystallite size $\left(d_{\mathrm{WH}}\right)$ and micro strains $\left(\varepsilon_{\mathrm{WH}}\right)$ for the $\alpha-\mathrm{Fe}_{2} \mathrm{O}_{3}$-based samples. \\
Samples & $d_{\mathrm{WH}} / \mathrm{nm}$ & $-(3.66 \pm 0.50) \cdot 10^{-3}$ \\
NF-600 & $17.7 \pm 1.3$ & $-(3.11 \pm 0.19) \cdot 10^{-3}$ \\
NF-600/10 & $19.4 \pm 0.6$ & $-(3.01 \pm 0.58) \cdot 10^{-3}$ \\
NF-600/25 & $19.7 \pm 1.8$ & $-(2.65 \pm 0.03) \cdot 10^{-3}$ \\
NF-600/100 & $21.2 \pm 0.1$ & $-(5.38 \pm 1.13) \cdot 10^{-3}$ \\
NF-25/600 & $12.4 \pm 1.4$ & \\
\hline
\end{tabular}



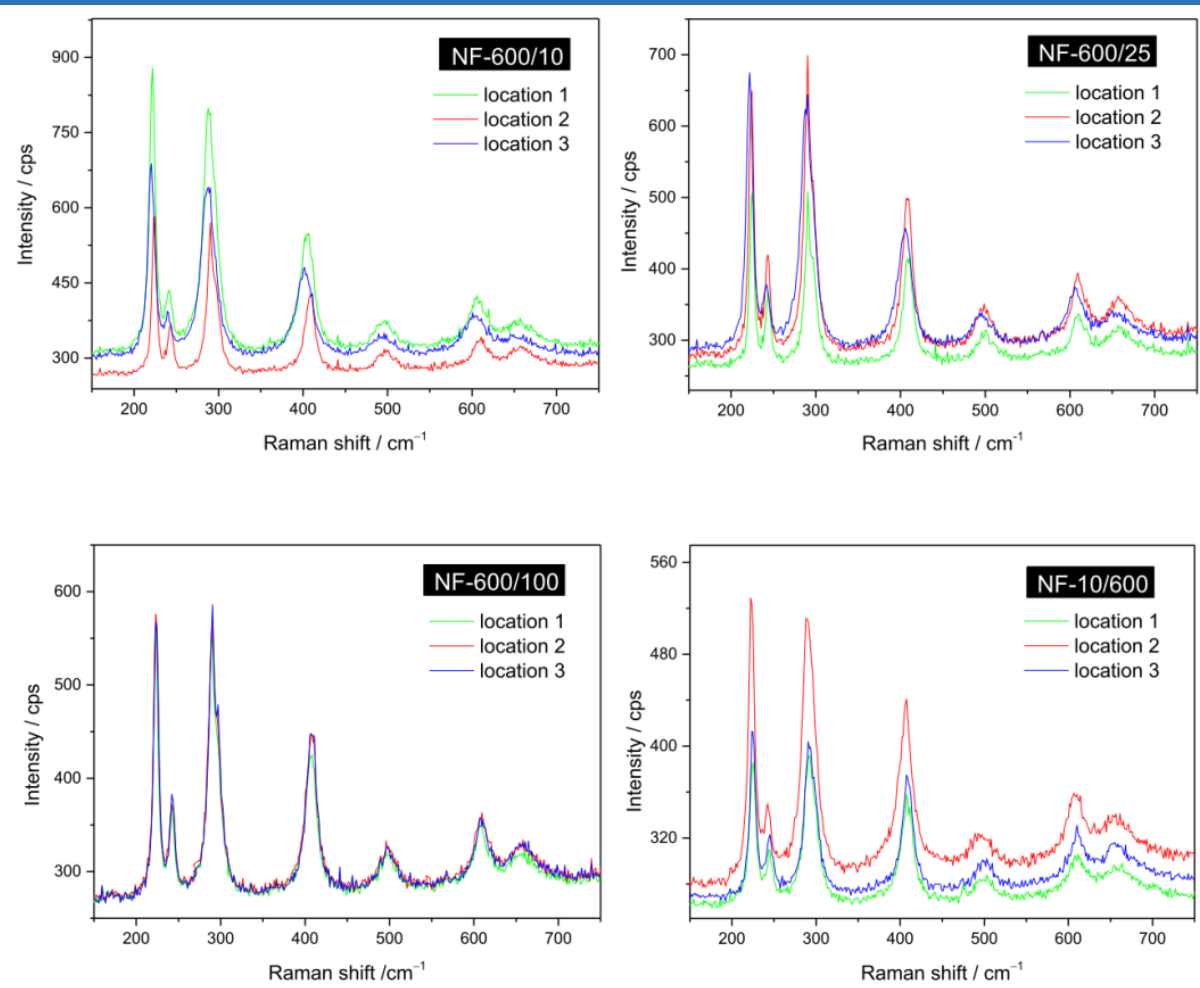

Figure S13. Micro-Raman spectra, as measured at different random locations, within each sample (grating: 1800 lines $\mathrm{mm}^{-1}$ ). For the peak assignation, refer to Table S2.

Table S2. Frequency position, symmetry and assignation of the main spectral features in the Raman spectrum of hematite. Hematite, with rhombohedral unit cell, belongs to the crystal space group $\mathrm{D}_{\mathrm{d} 6}^{3}$. According to space group symmetry and factor group analysis, its normal modes at the Brillouin zone center include seven Raman-active vibrations $\left(2 A_{1 \mathrm{~g}}+5 E_{\mathrm{g}}\right)$, six IR-active vibrations $\left(2 A_{2 \mathrm{u}}+4 E_{\mathrm{u}}\right)$, and five optically silent vibrations $\left(2 A_{1 \mathrm{u}}+3 A_{2 \mathrm{~g}}\right) .{ }^{\mathrm{S} 7}$

$\begin{array}{cccccc}\text { Peak } & \text { Position } / \mathrm{cm}^{-1 \text { S8-S11 }} & \text { FWHM } / \mathrm{cm}^{-1} \text { S9(a) } & \text { Symmetry } & \text { Mode }^{\mathrm{S8}} & \text { Moving ion }^{\mathrm{S} 7} \\ 1 & 224-227 & 4.9 & A_{\mathrm{gg}}(1) & \mathrm{Fe}-\mathrm{O} \text { sym. stretching } & \text { Iron } \\ 2 & 243-247 & 6.0 & E_{\mathrm{g}}(1) & \mathrm{Fe}-\mathrm{O} \text { sym. bending } & \text { Iron } \\ 3 & 292-300 & 8.5 & E_{\mathrm{g}}(2), E_{\mathrm{g}}(3) & \mathrm{Fe}-\mathrm{O} \text { sym. bending } & \text { Iron } \\ 4 & 408-413 & 11.6 & E_{\mathrm{g}}(4) & \mathrm{Fe}-\mathrm{O} \text { sym. bending } & \text { Oxygen } \\ 5 & 495-500 & 21.2 & A_{1 \mathrm{~g}}(2) & \mathrm{Fe}-\mathrm{O} \text { sym. stretching } & \text { Oxygen } \\ 6 & 600-612 & 14.4 & E_{\mathrm{g}}(5) & \mathrm{Fe}-\mathrm{O} \text { sym. bending } & \text { Oxygen } \\ 7 & 657-660 & & E_{\mathrm{u}}(1) & & \end{array}$

$8 \quad 1310-1320$

(a) For natural hematite, laser power at the sample surface: $0.7 \mathrm{~mW}$

Two-magnon scattering ${ }^{(\mathrm{b})}$

(b) The two-magnon scattering arises from the interaction of two magnons created on antiparallel close spin sites. ${ }^{\text {S9-S11 }}$ 

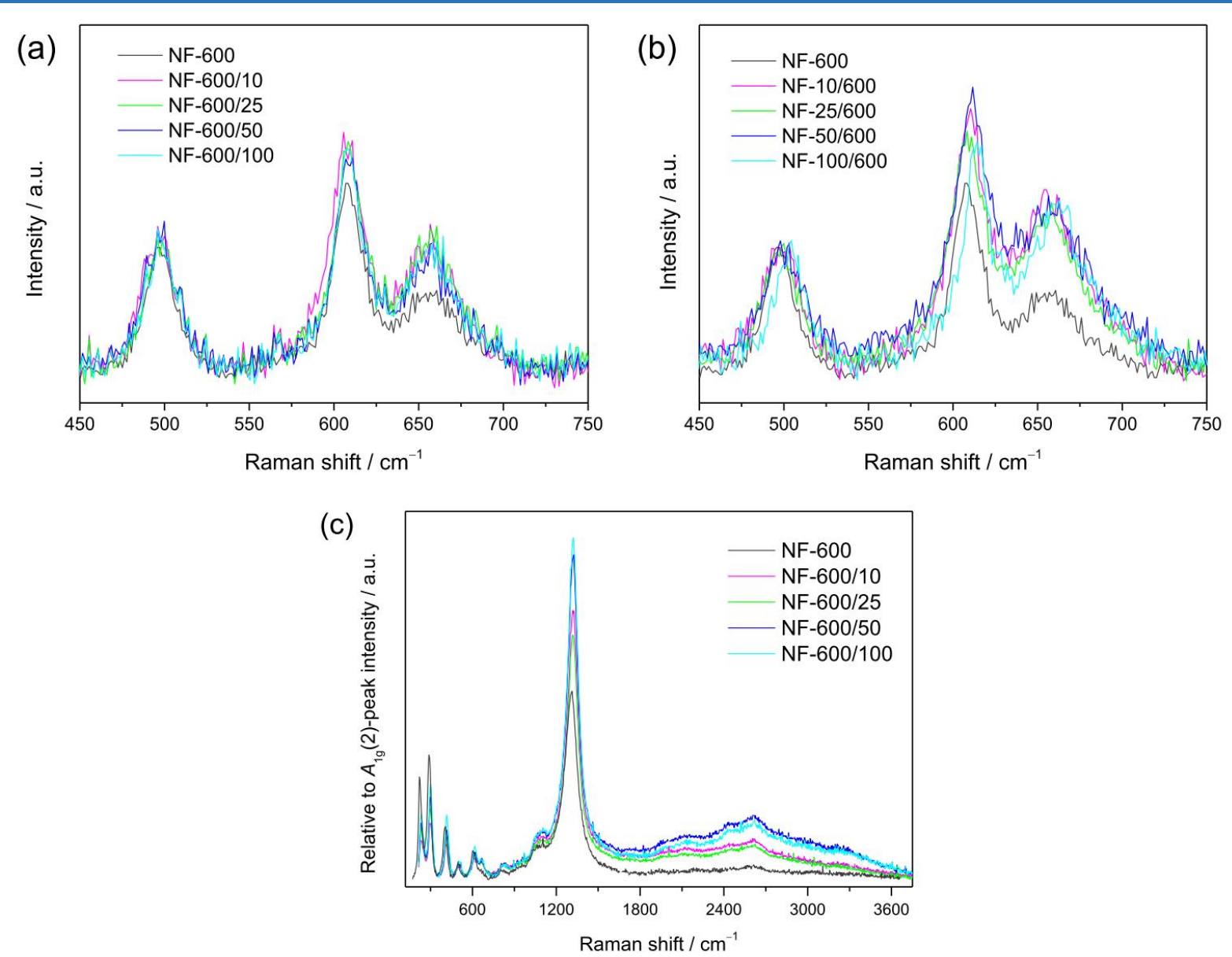

Figure S14. (a,b) Higher frequency region of the micro-Raman spectra in samples (a) NF-600/N and (b) NF-N/600 after background subtraction and normalization to the $A_{1 \mathrm{~g}}(2)$ peak maximum intensity. (c) Micro-Raman spectra of the samples NF-600/N recorded by the use of a 600 lines $\mathrm{mm}^{-1}$ grating and normalized to the $A_{1 \mathrm{~g}}(2)$ peak maximum intensity. For the peak assignation, see Table S2. 

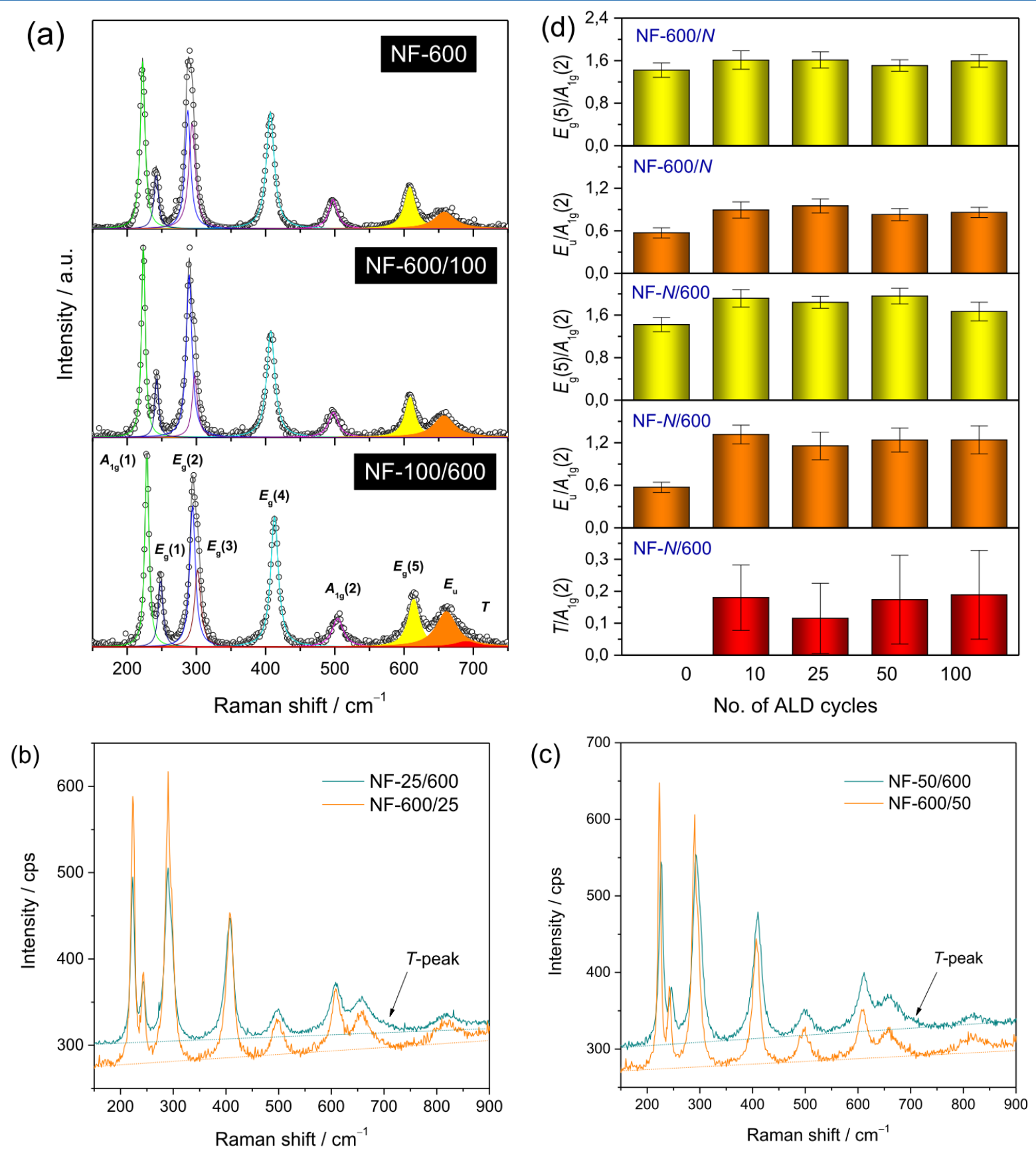

Figure S15. (a) Fits of the micro-Raman spectra to Lorentzian bands. (b,c) Comparison between as-measured spectra of samples NF-600/N and NF-N/600 for (b) $N=25$ and (c) $N=50$ ). (d) Relative intensities of the higher-frequency Raman modes. 

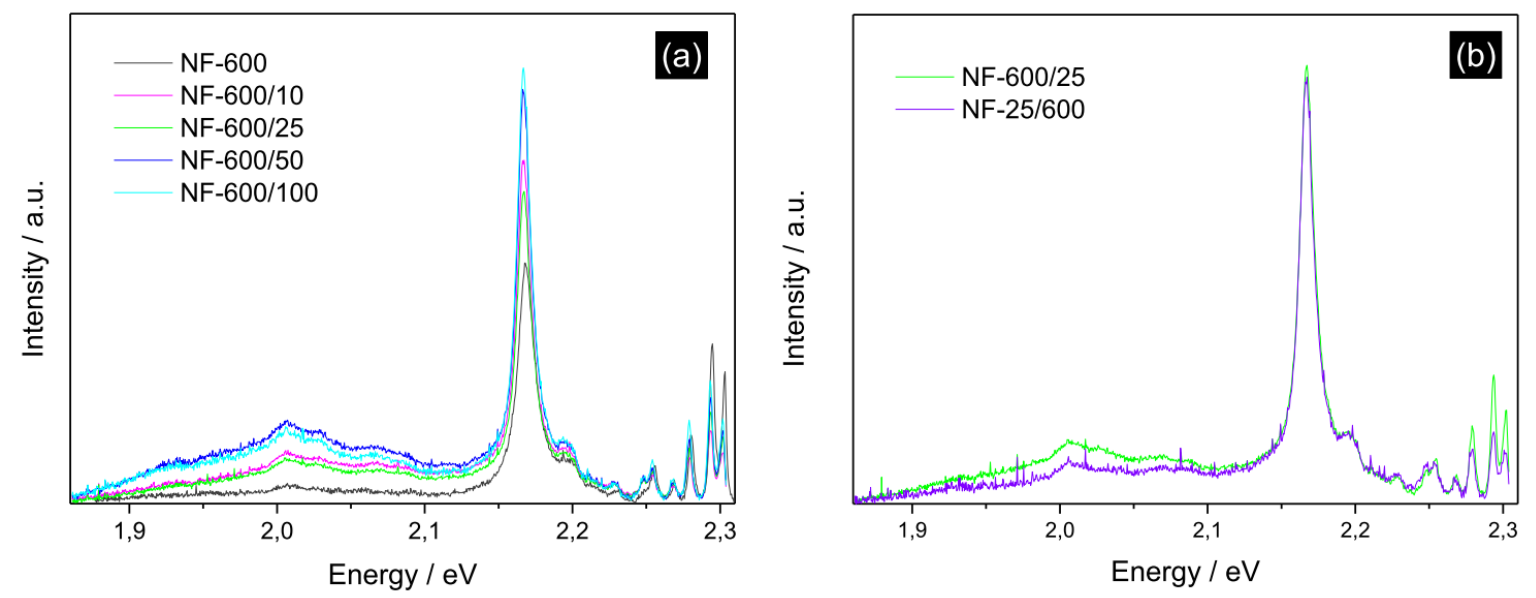

Figure S16. (a,b) Photoluminescence spectra. (a) Effect of the variation of $N$ in samples NF-600/N and (b) comparison between samples NF-600/N and NF-N/600 for a given $N$ value. 


\section{Magnetic Properties}
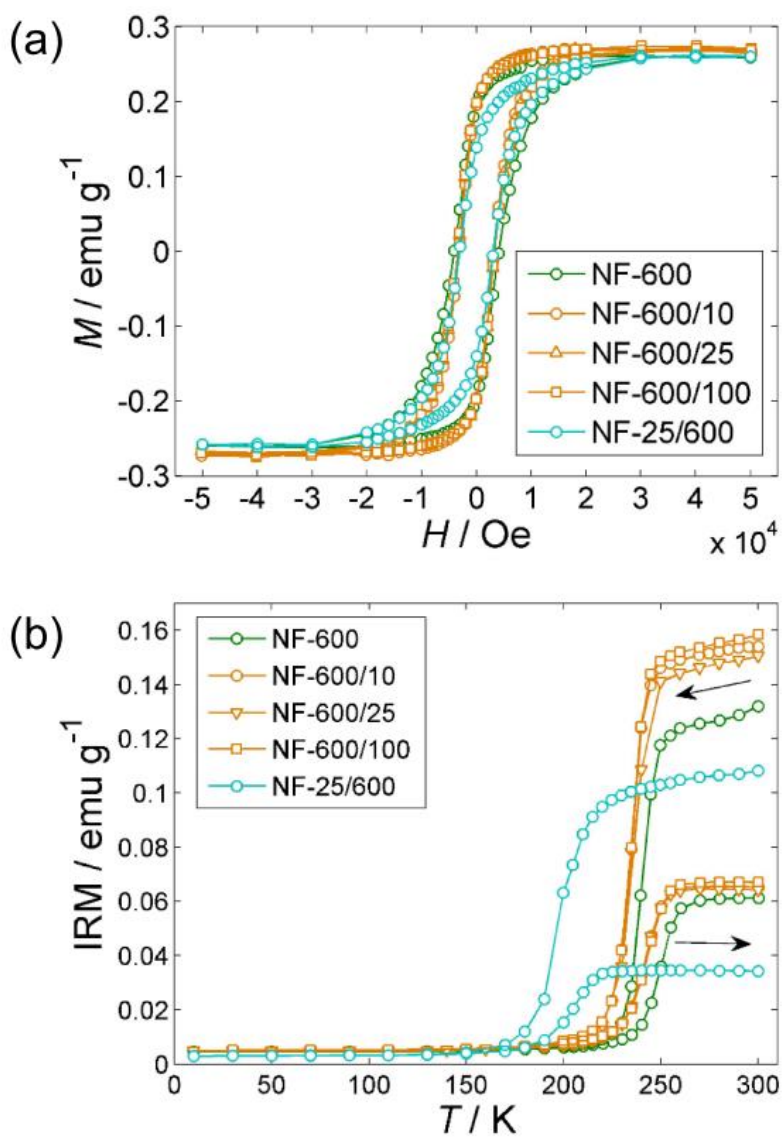

Figure S17. Full-range plots of the magnetic behavior of the hematite nanofibers. (a) Magnetization isotherms (hysteresis loops) at $300 \mathrm{~K}$ after subtraction of the paramagnetic contribution. (b) Thermal behavior of the isothermal remnant magnetization (IRM) during cooling to $10 \mathrm{~K}$ and warming to $300 \mathrm{~K}$.

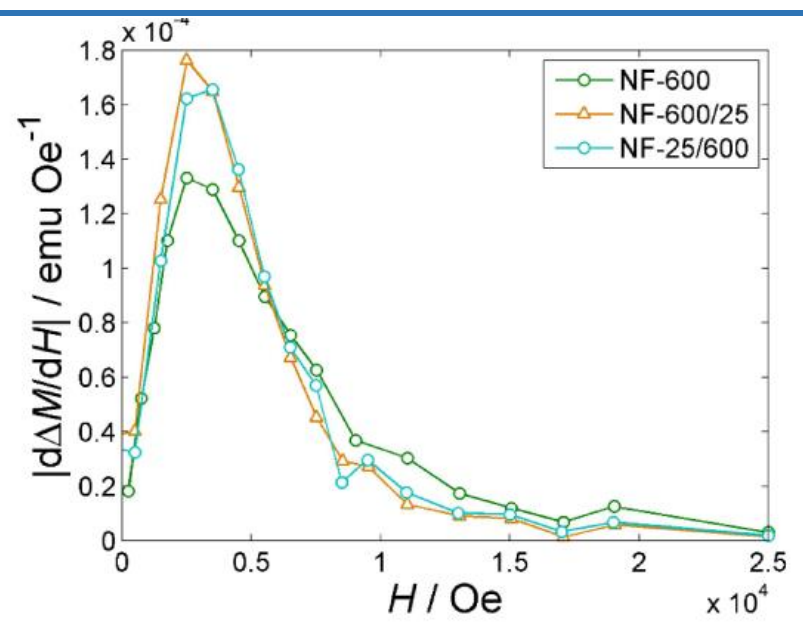

Figure S18. Microcoercivity spectrum of selected hematite nanofibers at $300 \mathrm{~K}$ calculated using the $\Delta M$ method. ${ }^{\text {s12 }}$ 


\section{- REFERENCES}

(S1) Bachmann, J.; Zierold, R.; Chong, Y. T.; Hauert, R.; Sturm, C.; Schmidt-Grund, R.; Rheinländer, B.; Grundmann, M.; Gösele, U.; Nielsch, K. A practical, self-catalytic, atomic layer deposition of silicon dioxide. Angew. Chemie - Int. Ed. 2008, 47, $6177-6179$.

(S2) Hytch, M. J. Analysis of variations in structure from high-resolution electron microscope images by combining real space and Fourier space information. Microsc. Microanal. Microstruct. 1997, 8, 41-57.

(S3) Rouvière, J. L.; Sarigiannidou, E. Theoretical discussions on the geometrical phase analysis. Ultramicroscopy 2005, 106, 1-17.

(S4) Bindu, P.; Thomas, S. Estimation of lattice strain in ZnO nanoparticles: X-ray peak profile analysis. J. Theor. Appl. Phys. 2014, 8, 123-134.

(S5) Khorsand Zak, A.; Abd. Majid, W. H.; Abrishami, M. E.; Yousefi, R. X-ray analysis of ZnO nanoparticles by Williamson-Hall and size-strain plot methods. Solid State Sci. 2011, 13, 251-256.

(S6) Cullity, B. D. Elements of X-Ray Diffraction. 2nd Edition, Addison-Wesley Publishing Company Inc., Phillippines 1978.

(S7) Chernyshova, I. V.; Hochella, M. F.; Madden, A. S. Size-Dependent Structural Transformations of Hematite Nanoparticles. 1. Phase Transition. Phys. Chem. Chem. Phys. 2007, 9, 1736-1750.

(S8) Legodi, M. A.; de Waal, D. The preparation of magnetite, goethite, hematite and maghemite of pigment quality from mill scale iron waste. Dye. Pigment. 2007, 74, 161-168.

(S9) de Faria, D. L. A.; Venâncio Silva, S.; de Oliveira, M. T. Raman microspectroscopy of some iron oxides and oxyhydroxides. J. Raman Spectrosc. 1997, 28, 873-878.

(S10) Bersani, D.; Lottici, P. P.; Montenero, A. Micro-Raman Investigation of Iron Oxide Films and Powders Produced by SolGel Syntheses. J. Raman Spectrosc. 1999, 30, 355-360.

(S11) de Faria, D. L. A.; Lopes, F. N. Heated goethite and natural hematite: can Raman spectroscopy be used to differentiate them? Vib. Spectrosc. 2007, 45, 117-121.

(S12) Ponti, A.; Ferretti, A. M.; Capetti, E.; Spadaro, M. C.; Bertoni, G.; Grillo, V.; Luches, P.; Valeri, S.; D’Addato, S. Steering the magnetic properties of $\mathrm{Ni} / \mathrm{NiO} / \mathrm{CoO}$ core-shell nanoparticle films: The role of core-shell interface versus interparticle interactions. Phys. Rev. Mater. 2017, 1, 036001. 